\title{
Antitubercular Therapy in Breast Tuberculosis and in Diagnostic Dilemma with Chronic Recurrent Mastitis: Bangladesh Perspective
}

\section{Introduction}

TB is a global emergency declared by The World Health Organization in 1993. According to WHO, 50 per cent of Bangladesh's adult population is infected by TB bacilli. Estimated incidence of TB in 2005 in Bangladesh was 227 per 100,000 population. Among 22 high-burden countries, Bangladesh ranks sixth. More than 300,000 being identified as new cases, with an estimated 70000 TB-related deaths occurring a year. A total of 102,785 new smear-positive cases were detected between July 2006 and June 2007. ${ }^{1}$ Though pulmonary tuberculosis is endemic but breast tuberculosis not reported so much in this region with a high incidence of tuberculosis infection. Relative resistance of breast tissue to the mycobacterium tuberculosis is one of the reasons for this. Mammary gland tissue offers resistance to the survival and multiplication of the tubercle bacillus, like the spleen and skeletal muscle, making it comparatively uncommon site for tuberculosis. ${ }^{2}$

Most commonly tubercular Infection of the breast occur through lymphatic, it may spread also through direct spread from contiguous structures. Haematogenous route is rare, usually seen In immunocompromised patients. ${ }^{2}$ Mammary tuberculosis is not uncommon disease in our country. There is very high incidence of pulmonary tuberculosis in the subcontinent as a result different forms of extra-pulmonary tuberculosis are also comparatively high in this region. ${ }^{3}$ Careful history taking and physical examination along with FNAC will lead to the diagnosis. ${ }^{4}$

Breast TB is paucibacillary. There are wide range of clinical presentation, radiological features and even microbiological presentation of Breast tuberculosis. Like pulmonary tuberculosis diagnostic tests microscopy, culture and polymerase chain reaction techniques do not have the same diagnostic utility in mammary tuberculosis. ${ }^{5}$

Thus breast TB is often diagnosed wrongly as non-specific abscess, granulomatous inflammation or carcinoma. ${ }^{6,7}$ Recurrence is a major factor in Mammary tuberculosis. Due to development of local recurrence, surgeons performed mastectomies to treat mammary tuberculosis for most of the cases before the discovery of antituberculosis drugs. According to Wilson et al. ${ }^{8}$ simple mastectomy was the treatment of choice.

Medical treatment consisting of a four drug regimen now forms the basis of treatment, with Limited surgical intervention. Usually surgery done in case of mammary tuberculosis is biopsy to help in diagnosis, aspiration of cold abscesses, and excision of residual sinuses and masses. ${ }^{2,9}$

\section{Methods}

Retrospective data of 68 consecutive patients with patients receiving Antitubercular therapy was analyzed and information regarding clinical presentation, cytology \& histopathology report and management was noted. Antitubercular therapy remained mainstay
Volume 3 Issue 3 - 2016

\author{
Laila Shirin,' Saifuddin Ahmed² \\ 'Department of Surgical Oncology, National Institute of Cancer \\ Research and Hospital, Bangladesh \\ ${ }^{2}$ Department of Surgical oncology, Bangabandhu Sheikh Mujib \\ Medical University, Bangladesh
}

Correspondence: Dr Laila Shirin, Department of surgical Oncology, National Institute of Cancer Research \& Hospital, Mohakhali, Dhaka, Bangladesh, Tel +880I819296903, Email dr.Ishirin@gmail.com

Received: July 17, 2016 | Published: August 24, 2016

of treatment with surgical intervention done in some cases when required.

Objective of the study was to report the clinical spectrum, investigative profile, diagnostic dilemma and management of patients with Antitubercular therapy in patients with breast tuberculosis and also in chronic recurrent cases (such as chronic granulomatous inflammation \& chronic inflammation of breast) where traditional treatment was not responded.

\section{Results}

In this study $48 \%$ was $20-30$ years of age and total $72 \%$ was within 20-40years of age. (Table 1) Out of them $88 \%$ were married and $12 \%$ unmarried. In the study population presentation was a solitary breast lump in $19(28 \%)$ patients, breast lump with axillary lymphadenopathy in $6(8.82 \%)$. Fourteen $(21 \%)$ patients presented with generalized breast swelling, tenderness with ipsilateral axillary lymphadenopathy. Two (2.94\%) presented with breast abscess and axillary lymphadenopathy, abscess only $2(2.94 \%)$, recurrent breast abscess in 5(7.35\%) and non healing Ulcer presented in 2(2.94\%) cases. Breast lump mimicking Antibioma in 08(11.76\%) cases. Breast carcinoma suspected in six cases; by breast lump in 3(04.41\%) cases, by bloody nipple discharge in $2(2.94 \%)$ cases and by nipple retraction in one case. Patients presented with axillary sinus in $2(2.94 \%)$ and breast sinus in $2(2.94 \%)$ cases (Table 2 ).

Twenty seven $(39.70 \%)$ cases were secondary to tuberculosis in other sites, mostly $22(32.35 \%)$ from tuberculous axillary lymphadenitis, although $14(20.58 \%)$ case presented with Generalized Breast swelling, tenderness with axillary lymphadenopathy investigations proved to be tuberculosis of axillary lymph node only but pain and swelling of the breast improved after ATT. Five $(7.35 \%)$ cases were secondary to pulmonary tuberculosis. In the study group $\mathrm{H} / \mathrm{O}$ pulmonary $\mathrm{TB}$ was present in $5(7.35 \%)$ and general sign symptom of TB in $9(13.23 \%)$ cases. At the time of diagnosis $3(4.41 \%)$ were pregnant and $11(16.17 \%)$ were lactating. H/O taking Anti TB was present in $2(2.94 \%)$ and $\mathrm{H} / \mathrm{O}$ previous surgery for breast abscess in $5(7.35 \%)$ cases. 
The cytological findings of Epitheloid cells, lymphocyte, granuloma, caseous necrosis were present in $79 \%$ cases and no typical caseous necrosis diagnostic of TB in $16 \%$ cases. There were no granuloma, no caseous necrosis present in $4.4 \%$ cases (Table 3). FNAC exclude malignancy in the suspected two cases of breast lump. Another two cases report was not conclusive after FNAC and malignancy was excluded by excision biopsy. Histopathology report of those cases was chronic abscess. On cytology from bloody nipple discharge no malignant cell present \& FNAC exclude malignancy in those cases.

Surgical Treatment undertaken All patients received Antitubercular therapy of four drugs regime of 9 month $\&$ in some cases up to 1year. Out of 68patients surgical intervention done on $36(53 \%)$ patients. No operation undertaken in 32(47\%)patients. Types of operation included excision and biopsy of mass in 14(20.58\%) patients, Out of 14cases excision of breast lump done in 3 cases of antibioma , 6cases of breast lump without caseous necrosis and 5 cases of residual breast lump after ATT needs excision. Excision and biopsy of axillary lymph node done in 6patients, Four cases of residual axillary lymph node after ATT \& two cases of nonspecific lymphaenitis on FNAC needs excision. I/D of abscess with biopsy of abscess wall in $13 \%$ and simple mastectomy in one patient (Table 4). On histopathology $90.7 \%$ were reported as $\mathrm{TB}, 4.6 \%$ as chronic granulomatous inflammation and another $4.6 \%$ as chronic inflammation only (Table 5).

All patients were followed up at 2 month, 6month, 9month \& 1year. Extension of antitubercular therapy from 9month extended up to 12 months was required in some patients on the basis of their slow clinical response. Complete resolution was obtained in 67patients. One patient did not respond to antitubercular therapy and repeated surgical attempt was failed to cure the patient and simple mastectomy was required.

Table I Age in years $(n=68)$

\begin{tabular}{lll}
\hline Age in years & Number & $\mathbf{\%}$ \\
\hline $20-$ Oct & 10 & 14.7 \\
$20-30$ & 33 & 48.52 \\
$30-40$ & 16 & 23.52 \\
$40-50$ & 6 & 8.82 \\
$50-60$ & 3 & 4.41 \\
Total & 68 & 100 \\
\hline
\end{tabular}

Table 2 Clinical presentation of the study population $(n=68)$

\begin{tabular}{llc}
\hline Clinical presentation & Number & \% \\
\hline Breast lump & 19 & 27.94 \\
Breast lump with axillary lymphadenopathy & 6 & 8.82 \\
Generalized Breast swelling,tenderness with axillary lymphadenopathy & 14 & 20.58 \\
Breast abscess with axillary lymphadenopathy & 2 & 2.94 \\
Breast abscess & 2 & 2.94 \\
Recurrent Breast abscess & 5 & 7.35 \\
Nonhealing Ulcer & O2 \\
Antibioma & 8 & 2.94 \\
Breast lump mimicking breast carcinoma & 3 & 11.76 \\
Nipple retraction mimicking breast carcinoma & 3.41 \\
Bloody nipple discharge & 1 & 1.47 \\
Axillary sinus & 2 & 2.94 \\
Breast sinus & 2 & 2.94 \\
Total & 2 & 2.94 \\
\hline
\end{tabular}


Table 3 Result of FNAC $(n=68)$

\begin{tabular}{lll}
\hline Report & Number & $\%$ \\
\hline Epitheloid cells, lymphocyte, granuloma, caseous necrosis & 54 & 79.41 \\
Epitheloid cells, lymphocyte, granuloma, no caseous necrosis & 11 & 16.18 \\
Chronic lymphocyte, no granuloma, no caseous necrosis & 3 & 4.41 \\
Total & 68 & 100 \\
\hline
\end{tabular}

Table 4 Surgical Treatment undertaken $(n=68)$

\begin{tabular}{lll}
\hline Surgical Treatment & Number & \% \\
\hline Excision and biopsy of breast lump & 14 & 20.58 \\
Excision and biopsy of axillary lymph node & 6 & 8.82 \\
I/D of abscess with biopsy of abscess wall & 9 & 13.23 \\
Excision and biopsy of sinus tract & 4 & 5.88 \\
Excision and biopsy of ulcer margin & 2 & 2.94 \\
Mastectomy & 1 & 1.47 \\
No surgery & 32 & 47.05 \\
\hline
\end{tabular}

Table 5 Diagnosis after histopathology $(n=68)$

\begin{tabular}{llc}
\hline Diagnosis & Number & $\%$ \\
\hline Chronic granulomatous inflammation tuberculosis & 62 & 90.76 \\
Chronic granulomatous inflammation & 3 & 4.61 \\
Chronic inflammation & 3 & 4.61 \\
Total & 68 & 100 \\
\hline
\end{tabular}

\section{Discussion}

Breast tuberculosis (TB) is a rare disease. It is less common in western countries with an incidence of less than $0.1 \%$ of all breast lesions whereas in TB endemic countries estimated incidence is $4 \%$ of all breast lesions. ${ }^{2,10}$ It typically affects young, lactating and multiparous women. Most commonly presented as painless breast mass or an abscess or sinus which is usually unilateral. ${ }^{2,10}$

Studies have shown that a high proportion of breast TB patients do not present with pulmonary or systemic symptoms. Breast TB can either be primary or secondary. In primary breast TB breast lesion is the only manifestation of TB. Where there is a demonstrable focus of TB else where it is secondary. ${ }^{2,10}$ Primary breast TB is rare. Most cases are considered secondary even if no primary focus can be found. ${ }^{2}$ Breast tuberculosis usually affects the younger women of child bearing age between $20-40$ years. ${ }^{11,12}$ In the study by Khanna et al. ${ }^{12}$ the mean age was 33.71 year. In our present series $49(72 \%)$ of patients were within the age range of 20-40years. Mean age was 29.3, age range 16 to 58 and in between $26-35 y$ rs by Deepa $\mathrm{H}$ et al. ${ }^{13}$

Breast tissue shows periodic changes with menstruation and more liable to trauma and infection within the reproductive age group. During pregnancy and lactation breast tissue engorged with blood vessels and dilated ducts. Cracked nipple during lactation and inoculation of bacteria from baby's mouth making them more susceptible to tubercular infection. ${ }^{11}$ Shinde et al. found $7 \%$ of their patients to be lactating at the time of presentation, and $30 \%$ of the women were lactating at presentation by Khanna et al. ${ }^{12}$ patients were lactating $(35.7 \%)$ at the time of diagnosis by Deepa $\mathrm{H}$ et al. ${ }^{13}$ Banerjee et al. ${ }^{14}$ reported $33 \%$ of their patients to be lactating..$^{14}$ At the time of presentation $11 \%$ the women were lactating in our series.

In the study population $36(53 \%)$ cases were presented with breast lump. Presentation was a solitary breast lump in $19(28 \%)$ patients, breast lump with axillary lymphadenopathy in $6(8.82 \%)$. Breast lump mimicking Antibioma in $08(11.76 \%)$ cases. Breast carcinoma suspected by breast lump in $3(04.41 \%)$ cases. Presentation was breast abscess in 9 cases. Two $(2.94 \%)$ presented with breast abscess and axillary lymphadenopathy, abscess only $2(2.94 \%)$ and recurrent breast abscess in 5(7.35\%) cases.

A lump is the commonest clinical presentation of breast tuberculosis. ${ }^{2,3,15}$ It coincides with our finding where presentation was a solitary breast lump in 30(60\%) patients. Upper outer quadrant was most frequently involved in $29(58 \%)$ of patients. A slowly growing solitary painless mass may be indistinguishable from carcinoma breast, being irregular, hard, sometimes may be fixed to the surrounding 
skin or chest wall.,16 Inflammation with abscess, ulceration, sinus or diffuse mastitis also occurs in significant proportion. Deepa $\mathrm{H}$ et al. ${ }^{13}$ in their study found breast abscess in $(64.3 \%)$ cases. Breast lump mimicking carcinoma breast in $14.3 \%$ and fibrocystic breast disease in $21.4 \%$ cases.

The commonest mode of spread to the breast is lymphatic \& the axillary lymph nodes. Direct extension from the chest wall (pleura or ribs) or and by inoculation of broken skin or duct openings can occur. Haematogenous spread is comparatively rare, Jah $\mathrm{H}$ et al. ${ }^{17}$ Studies reported axillary lymph node enlargement in 50\%-70\% cases of mammary tuberculosis which explained the retrograde lymphatic extension from axillary lymph nodes to breast. ${ }^{18}$

In study conducted by Jalali et al. ${ }^{15}$ Thirty-two $(64 \%)$ cases were secondary to tuberculosis in other sites, from tuberculous axillary lymphadenitis in $(40 \%)$ cases. Study by Sharma et al. ${ }^{19}$ showed that same side axillary lymph node involvement was present in 3 cases $(43 \%)$. Vassilakos ${ }^{20}$ also supporting that axillary node involvement occurs in 50 to 75 per cent of cases of tuberculosis mastitis. In our series Twenty seven $40 \%$ cases were secondary to tuberculosis in other sites; mostly $32 \%$ from tuberculous axillary lymphadenitis five $(7.35 \%)$ cases were secondary to pulmonary tuberculosis.

FNAC has been found to be of very effective in diagnosing of breast tuberculosis. Tuberculous mastitis can be diagnosed reliably by cytological evidence of epithelioid granulomas, Langhans' giant cells, and lymphohistocytic aggregates and presence of caseous necrosis. ${ }^{21}$ Reported by Kakker et al. ${ }^{22} 73 \%$ of breast tuberculosis in can be diagnosed on the basis of FNAC findings of epithelioid cell granulomas with caseous necrosis. Fine-needle aspiration cytology (FNAC) is useful in diagnosis of breast lumps with or without nodes. ${ }^{21-23}$ Acid-fast bacilli (AFB) is not mandatory for diagnosis.

Failure to identify necrosis in FNAC does not exclude tuberculosis..$^{23}$ As in FNAC small quantity of the sample examined so there may be every possibility to fail to demonstrate necrosis. So in these cases only epitheloid granulomas identifies which makes a mistaken diagnosis of granulomatous inflammation. ${ }^{2,23}$ According to Tewari et al. ${ }^{2}$ FNAC can diagnose breast TB in as many as three quarters of cases. On the other hand Khanna et al. ${ }^{12}$ stated that $100 \%$ reliable in diagnosing tuberculosis can be made by FNAC or histology.

In our present series FNAC confidently diagnosed TB in $79 \%$ cases. However On histopathology $90.7 \%$ were reported as TB, $4.6 \%$ as chronic granulomatous inflammation and another $4.6 \%$ as chronic inflammation only. FNAC and excision biopsy exclude all the cases of suspected malignancy and in this series there was confusion in differentiating tuberculosis from malignancy by FNAC in two cases but malignancy excluded histologically. This may be due to the fact that our pathologists are oriented in diagnosis of tuberculosis as it is a tuberculosis endemic country. There were several reports where FNAC was not conclusive \& microscopically cells were suspicious of malignancy and lumpectomy or even simple mastectomy was done on the basis of suspected malignancy. On subsequent histopathological examination tubercular mastitis was confirmed.

Granulomatous mastitis forms an important histological differential diagnosis of tuberculosis and other differential diagnoses include plasma cell mastitis and actinomycosis ${ }^{20}$ Study suggested that In majority of cases the diagnosis is established only after histopathological evaluation of the biopsy. Though the gold standard in establishing the diagnosis of tuberculosis is demonstration of the causative organism Mycobacterium tuberculosis in Z-N stain or in culture it is often not possible to found it on culture. So an excision biopsy is strongly suggested to rule out other diagnoses like sarcoidosis, fungal infections, ductular ectasia, and a coexisting malignancy, ${ }^{2,23,25}$

The diagnosis of granulomatous mastitis is made histologically by identifying non-caseating granulomatous inflammation with giant cells, leucocytic infiltrations, foamy macrophages and abscesses which overlap with breast TB. Thus to differentiate granulomatous mastitis from $M$. tuberculosis is very difficult \& sometimes they can't be identified separately at all. ${ }^{26,27}$ Out of 68patients surgical intervention done on $36(53 \%)$ patients. No operation undertaken in $32(47 \%)$ patients. Types of operation included excision and biopsy of mass in $14(20.58 \%)$ patients, excision and biopsy of axillary lymph node in $9 \%$ patients, I/D of abscess with biopsy of abscess wall in $13 \%$ and simple mastectomy in one patient.

Khanna et al. ${ }^{12}$ stated that complete resolution occur in 42patients (out of 52) after ATT, two had needed mastectomy. Medical treatment consisting of a four drug regimen forms the basis of treatment. Surgical intervention is reserved for aspiration of cold abscesses, and excision of residual sinuses and masses. ${ }^{9,12,14}$ In refractory cases with destruction of the breast, simple mastectomy may be performed. The recommended treatment for granulomatous mastitis is completes resection or open biopsy with corticosteroid therapy ${ }^{28}$ Granulomatous mastitis can recur in up to $50 \%$ cases despite appropriate therapy. Granulomatous mastitis is sometimes also complicated by abscess formation, fistulae, and chronic suppuration. ${ }^{24}$ Some patients have had relapses in the form of chronic mastitis even after excisional biopsies. $^{12}$

In $3(4.6 \%)$ cases of Chronic granulomatous inflammation there was recurrence after excision biopsy. Histopathology failed to identify tuberculosis. Mt test was positive. We use ATT with success. In three cases $3(4.6 \%)$ of Chronic recurrent inflammation, tuberculosis was try to established but repeated attempt of excision biopsy failed to prove tuberculosis. MT test and ESR was high and we decided to start ATT. Two cases were responded to ATT but in one case ultimately needed mastectectomy after failure of repeated surgery and ATT therapy.

Several documents were previously reported by authors in relation to tuberculosis mistaken as chronic granulomatous inflammation or abscess. Jah et al. ${ }^{17}$ in their study suggested that presence of chronic lymphocytic aggregate on FNAC or core biopsy may be considered sufficient to start ATT in high prevalence areas. They stated that a good therapeutic response to antitubercular chemotherapy may be the only way to confirm diagnosis in some cases. ${ }^{17}$ Chandanwale concluded that in developing countries like India, based on clinical history and features of epithelioid granulomas with or without necrosis and AFB negative, FNAC smears may be given a therapeutic trial of standard antitubercular drug. ${ }^{21}$

Sriram et al. ${ }^{27}$ reported a patient with breast abscess that was initially diagnosed and treated as granulomatous mastitis abscess. ${ }^{27}$ When patient not responded to therapy tuberculosis was concluded because she was migrated from a TB endemic country. They had started standard antituberculosis drugs with complete resolution of the breast abscess. ${ }^{27}$ Sen $\mathrm{M}$ et al. ${ }^{29}$ in their study emphasized that in the differential diagnosis of breast lesions breast TB should be included 
especially for patients from high risk populations and from endemic regions. They stated that all of their three cases reported had breast $\mathrm{TB}$, as they responded to antituberculosis treatment. ${ }^{29}$

Borens Feber B et al..$^{30}$ presented a case of primary mammary tuberculosis (MT), revealed by a voluminous tubercular abscess mimicking a recurrent pyogenic abscess. The diagnosis of tuberculosis was made on the basis of several risk factors such as female gender, lactation, young age, and origin from an endemic area and recurrence of abscess after adequate drainage..$^{30}$ They suggested that breast TB should be suspected when there is poor response to antibiotics used for treatment of breast abscess, especially in a young patient originating from a TB endemic country. ${ }^{30}$

Breast TB is a diagnosis of exclusion. It should be always suspected in poor response to antibiotic therapy of breast inflammation especially in patients of tuberculosis endemic countries. Trial of antituberculous therapy is recommended in case of a high clinical suspicion of TB. ${ }^{26}$ So the diagnosis of mammary tuberculosis was suggested by a combination of clinical suspicion and considering Bangladesh as tuberculosis endemic region.

Anti tubercular therapy was given in all 68patients. MT test were positive in those cases of chronic granulomatous inflammation and chronic inflammation. All patients were followed up for a period of 24 month. Extension of antitubercular therapy from 9month extending up to 12 months was required in some patients, when the clinical response was delayed. Complete resolution was obtained in 67(98.5\%) patients. One patient did not respond to antitubercular therapy and repeated surgical attempt was failed and simple mastectomy was required.

Similarly response was reported by Jalali et al. ${ }^{15}$ in their study where $96 \%$ of their patients responded to one year antituberculous treatment with complete disappearance of the lumps except two patients. In those cases excision of lump was done. ${ }^{15}$ No specific guidelines are available for the chemotherapy of breast tuberculosis., ${ }^{2,25}$

Patients received antituberculous therapy comprising rifampicin $450 \mathrm{mg}$, isoniazid $300 \mathrm{mg}$, pyrazinamide $1500 \mathrm{mg}$, and ethambutol $800 \mathrm{mg}$ per day for two months followed by rifampicin and isoniazid for another four months Extension of antitubercular therapy from 12 to 18 months was required in 18patients on the basis of their slow clinical response R Khanna et al., ${ }^{12}$ Hat well deepa et al., ${ }^{13}$ Salim Baharoon. ${ }^{25}$ Similar to our study some other studies reported DOTS Cat-III (2 HRZ + $7 \mathrm{HR})$ therapy for 9months due to lower relapse rate. ${ }^{2,8,25,31}$

In our series in addition to ATT therapy aspiration of abscess was done in 6patients, excision of lump in 14patients, drainage of abscess in two patients and one patient was subjected to simple mastectomy for disseminated disease. Out of 14 cases 3 cases of antibioma, 6 cases of breast lump without caseous necrosis and 5 cases of residual breast lump after ATT needs excision. Four cases of residual axillary lymph node \& two cases of nonspecific lymphaenitis on FNAC need excision. Presentation of breast tuberculosis is variable. Several studies suggested that though it is a rare disease it should always be suspected when evaluating cases of breast abscesses, fistulae or nodules, with poor response to classical non-tuberculosis antibiotic treatment. 32,33

Several articles from India also recommended that where tuberculosis is endemic, caseous necrosis with epitheloid cell granulomas even in absence of acid fast bacilli (AFB) considered sufficient for the diagnosis of tuberculosis. If patients don't responded to sufficient dose of anti-tuberculous treatment only in that case an alternative diagnosis can be considered. ${ }^{3,34}$

Finally diagnosis of tuberculosis of breast can be difficult due to variable clinical presentation, with wide range of differential diagnosis, failure to identify AFB in biopsy specimen. microbiological or histopathological confirmation is needed for diagnosis of breast TB. But sometimes clinical suspicion and strongly positive skin test may be the only available evidence. After failure of conventional management, antitubercular chemotherapy can achieve good response with cure in those cases.

\section{Conclusion}

In conclusion Breast tuberculosis is still prevalent is developing countries. Presentation is variable may with breast lump mimicking benign lesion, chronic breast abscess, granulomatous inflammation or malignancy of breast. Tuberculosis should always suspect in endemic area in recurrent $\&$ cases with delayed response to adequate treatment. FNAC or biopsy is required for definitive diagnosis. The anti-tubercular chemotherapy is the treatment of choice for breast tuberculosis and chronic recurrent breast inflammation with poor response to classical non-tuberculosis antibiotic treatment, especially in case of young women within reproductive age in a TB endemic country like Bangladesh.

\section{Acknowledgements}

None.

\section{Conflict of interest}

The author declares no conflict of interest.

\section{References}

1. WHO. World Health Organization Geneva Bangladesh; 2007.

2. Tewari M, Shukla HS. Breast tuberculosis: Diagnosis, clinical features \& management. Indian J Med Res. 2005;122(2):103-110.

3. Chulki S, Kulkarni D, Sulegaon R. Tuberculous mastitis: a case report. Journal of Evidence based Medicine and Healthcare. 2014;1(9):12121216.

4. Pramanik MAK, Haque MM, Rashid AM, et al. Tuberculosis of the breast: a case report. TJA. 2008;21(1):73-76.

5. Pai M, Riley LW, Colford JM. Interferon-gamma assays in the immunodiagnosis of tuberculosis: a systematic review. Lancet Infect Dis. 2004;4(12):761-776.

6. Morino GF, Rizzardi G, Gobbi F, et al. Breast tuberculosis mimicking other diseases. Trop Doct. 2007;37(3):177-178.

7. Kumar BNK, Pandya B, Das D, et al. Tubercular breast lesion a great mimicker of carcinoma breast. Nepalese Journal of Radiology. 2013;31(4):99-102.

8. Wilson TS, Macgregor JW. The diagnosis and treatment of tuberculosis of the breast. Can Med Assoc J.1963;89:1118-1124.

9. Romero C, Carreira C, Cereceda C, et al. Mammary tuberculosis: percutaneous treatment of mammary tuberculous abscess. Eur Radiol. 2000;10(3):531-533.

10. Akcakaya A, Eryilmaz R, Sahin M, et al. Tuberculosis of the breast. The Breast Journal. 2005;11(1):85-86. 
11. Shinde SR, Chandawarkar RY, Deshmukh SP. Tuberculosis of the breast masquerading as carcinoma: A study of 100 patients. World J Surg. 1995;19(3):379-381.

12. Khanna R, Prasanna G V, Gupta P, et al. Mammary tuberculosis: report on 52 cases. Postgrad Med J. 2002;78(921):422-424.

13. Deepa H, Vijay S, Jai PM, et al. Tubercular Mastitis Is Common In Garhwa Region of Uttarakhand: Clinico Athological Features Of 14 Cases. Journal of Clinical and Diagnostic Research. 2011;5(8):1569-1573.

14. Banerjee SN, Ananthakrishnan N, Mehta RB, et al. Tuberculous mastitis: a continuing problem. World J Surg. 1987;11(1):105-109.

15. Jalali U, Rasul S, Khan A, et al. Tuberculous mastitis. J Coll Physician Surg Pak. 2005;15(4):234-237.

16. Shinde SR, Chandawarkar RY, Deshmukh SP. Tuberculosis of the breas masquerading as carcinoma: A study of 100 patients. World J Surg. 1995;19(3):379-381.

17. Jah A, Mulla R, Lawrence FD, et al. Tuberculosis of breast: experience of a UK breast clinic serving ethnically diverse population. Ann R Coll Surg Engl. 2004;86(6):416-419.

18. Mehta G, Mittal A, Verma S. Breast tuberculosis- Spectrum and management. Indian J Surg. 2010;72(6):433-437.

19. Sharma PK, Babel AL, Yadav SS. Tuberculosis of breast (study of 7 cases). J Postgrad Med. 1991;37(1):24-26.

20. Vassilakos P. Tuberculosis of the breast. Cytological findings with fine needle aspiration. Acta Cytol. 1973;17(2):160-165.

21. Chandanwale SS, Buch AC, Gore CR, et al. Fine Needle Aspiration Cytology In Breast Tuberculosis: Diagnostic Difficulties - Study Of Eleven Cases. Indian J Tuberc. 2012;59(3):162-167.

22. Kakkar S, Kapila K, Singh MK, et al. Tuberculosis of the Breast: A cytomorphologic study. Acta Cytol. 2000;44(3);292-296.
23. Gupta D, Rajwanshi A, Gupta SK, et al. Fine needle aspiration cytology in the diagnosis of tuberculous mastitis. Acta Cytol. 2000;43(2):191-194.

24. Dhopade TG, Thakur BA, Kumar M. Primary tuberculosis of breast. Bombay Hospital Journal. 2009;46(1):86-87.

25. Baharoon S. Tuberculosis of the breast. Annals Thoracic Medicine. 2008;3(3):110-114.

26. De Sousa R, Patil R. Breast Tuberculosis or granulomatous mastitis: a Diagnotic dilemma. Annals of Tropical Medicine and Public Health. 2011;4(2):122-125.

27. Sriram KB, Moffatt D, Stapledon R. Tuberculosis infection of the breast mistaken for granulomatous mastitis: a case report. Cases J. 2008;1(1):273.

28. De Hertogh DA, Rossof AH, Harris AA, et al. Prednisone management of granulomatous mastitis. N Engl J Med. 1980;303(14):799-800.

29. Sen M, Gorpelioglu C, Bozer M. Isolated primary breast tuberculosis report of three cases and review of the literature. Clinics (Sao Paulo). 2009;64(6):607-610.

30. Borens Fefer B, Engohan Aloghe C, Noël JC, et al. et al. Primary mammary tuberculosis presenting as a voluminous abscess. JBR-BTR. 2008;91(2):54-57.

31. Kumar P, Sharma N. Primary MDR-TB of the breast. Indian J Chest Dis Allied Sci. 2003;45(1):63-65.

32. Wani I, Lone AM, Malik R, et al. Secondary tuberculosis of breast: case report. ISRN Surgery. 2011;ID529368:1-3.

33. Marinopoulods S, Lourantou D, Gatzionis T, et al. Breast tuberculosis; diagnosis, management and treatment. Int $J$ Surg Case Rep. 2012;3(11):548-550.

34. Surya Kant, R Dua, MM Goel. Bilateral tubercular mastitis. Lung India. 2007;24(3):90-93. 